\title{
Non-Participation in Child Health Days or Routine Immunization Services among Children under 5 Years of Age-Somaliland 2012
}

\author{
Charles Kinuthia ${ }^{1}$, Marie Therese Baranyikwa², Khadar M. Ahmed ${ }^{3}$, Awil Haji Ali ${ }^{4}$, \\ Assegid T. Kebede ${ }^{5}$, John Agbor ${ }^{2}$, David W. Brown ${ }^{6}$ \\ ${ }^{1}$ Consultant to the UNICEF Somalia Support Center, Nairobi, Kenya; ${ }^{2}$ UNICEF Somalia Support Center, Nairobi, Kenya; \\ ${ }^{3}$ Somaliland Ministry of Health, Hargeisa, Somaliland; ${ }^{4}$ UNICEF, Hargeisa, Somaliland; ${ }^{5}$ WHO, Somalia, Nairobi, Kenya; ${ }^{6}$ UNICEF, \\ New York, USA. \\ Email: dbrown@unicef.org
}

Received December $11^{\text {th }}, 2013$; revised January $13^{\text {th }}, 2014$; accepted January $20^{\text {th }}, 2014$

Copyright (C) 2014 Charles Kinuthia et al. This is an open access article distributed under the Creative Commons Attribution License, which permits unrestricted use, distribution, and reproduction in any medium, provided the original work is properly cited. In accordance of the Creative Commons Attribution License all Copyrights (C) 2014 are reserved for SCIRP and the owner of the intellectual property Charles Kinuthia et al. All Copyright (C) 2014 are guarded by law and by SCIRP as a guardian.

\section{ABSTRACT}

Background: After two decades of conflict, Somalia remains a fragile state where large scale displacement and inadequate access to functioning health services have left children vulnerable to morbidity and mortality from vaccine preventable disease. Children residing in the autonomous zone of Somaliland are similarly vulnerable to poor access to health care services. Following the conduct of a UNICEF-supported Multiple Indicator Cluster Survey in Somaliland during 2011 which captured information on immunization system performance, a survey was conducted to better understand the reasons for non-vaccination among children in Somaliland. Methods: The Somaliland Routine Immunization Non-Participation Survey (RINPS) was conducted in November 2012 to better understand the reasons for non-participation in both Child Health Days (CHDs) and Routine Immunization Services (RIS). RINPS was a cross-sectional household survey which used a two-stage sample design in order to obtain a representative sample of children 0 - 59 months of age residing in Somaliland. Thirty clusters were randomly selected from the 303 clusters for participation in the 2011 Somaliland MICS. A total of 867 children aged 0 - 59 months were identified and included in the analysis (overall response rate, 96\%). Findings: Caregivers lacked motivation to take their children to CHDs and for RIS and lacked information about why children need immunization. Routine vaccination or CHD cards were available for few children at the time of the survey. Almost one-fifth of children aged 0 - 59 months in Somaliland had not received at least one dose of vaccine for DTP, polio or measles vaccine from either CHD or RIS. Conclusion: Child Health Days have a role in at least some area of Somaliland to expand the reach of immunization services. The availability and delivery of sustainable routine immunization services need to be strengthened in Somaliland with a strong social mobilization program to raise awareness about the importance of routine immunization.

\section{KEYWORDS}

Immunization; Routine Immunization; Child Health Day; Survey; Somaliland

\section{Introduction}

More than 20 years of conflict in Somalia has resulted in a severely disrupted health care system, including the immunization delivery system. Since 2001, access and utilization of immunization services, based on coverage with the first and third dose, respectively of diphtheria, tetanus, and pertussis (DTP) containing vaccine, have been suboptimal, ranging from 40 - 60 percent of children vaccinated with DTP1 and $25 \%$ - $40 \%$ of children vaccinated with DTP3 [1]. Following a 2007-2008 assessment of the determinants of child health in Somalia conducted by the United Nations Children's Fund (UNICEF) and the World Health Organization (WHO), an 
accelerated young child survival strategy was developed by the respective agencies in collaboration with the authorities in Somalia that included the utilization of Child Health Days (CHDs) conducted twice per year to increase access to immunization services and other child health interventions in Somalia through nationwide outreach campaigns. The eighth round of CHDs was conducted in October 2012.

Recent survey data from Somaliland, an internationally recognized autonomous zone of Somalia, have reported that only about one third of children have access to immunization services (based on DTP1 coverage) and less than one-in-four children receive the recommended three doses of DTP [2]. As part of a comprehensive review of the Expanded Programme on Immunization (EPI) in Somaliland conducted by the Somaliland Ministry of Health (MOH), UNICEF/Somalia, and WHO/Somalia in November and December 2012, an assessment of participation in CHDs and reasons for non-participation in routine immunization services (RIS), were conducted to supplement information on routine immunization coverage levels from the UNICEF-supported 2011 Somaliland Multiple Indicator Cluster Survey (MICS) [2], and understand determinants of non-participation in both CHDs and RIS, reasons related to failure to immunize children 0 - 59 months of age, and the attitudes of caregivers that led children not to be taken for immunization against vaccine preventable diseases. This report describes the findings of the Somaliland Routine Immunization NonParticipation Survey (RINPS).

\section{Methods}

\subsection{Survey Instrument}

Data were collected by trained interviewers using a questionnaire which was developed and validated by the RINPS technical committee in conjunction with UNICEF, WHO and the US Centers for Disease Control and Prevention (CDC). Where possible the committee used existing questions and response categories from previously validated survey instruments. The questionnaire was divided into two panels: household and children 0 - 59 months of age. The household panel collected information on the number of residents in the household and, specifically the number of children 0 - 59 months of age. The panel on children 0 - 59 months of age was completed by caregivers and collected information on participation in the October 2012 CHD and RIS information (using both information from home-based vaccination records and caregiver recall) and asked about attitudes toward CHDs and RIS, types of services received, and the main source of information about both CHDs and RIS. Because cards were distributed as part of the Octo- ber 2012 CHD, examples of the CHD card and homebased routine immunization cards used in Somaliland were shown by surveyors to help caregivers differentiate between the two vaccination records.

\subsection{Sampling Strategy}

The RINPS was conducted in Somaliland from 6-30 November 2012. RINPS was a cross-sectional household survey which used a two-stage sample design in order to obtain a representative sample of children 0 - 59 months of age residing in Somaliland. The desired sample size (N) was estimated assuming a confidence level of $95 \%$ $(Z=1.96)$, a margin of error (e) of 0.05 , an estimated level of coverage (P) of $40 \%$, and a design effect of 2 using Equation (1).

$$
\mathrm{N}=\mathrm{Z}^{2} \cdot[\mathrm{P}(1-\mathrm{P})] / \mathrm{e}^{2}
$$

The sample size was increased by $10 \%$ to account for an assumed $90 \%$ response rate. The RINPS sample was selected based on probability proportional to size and included 30 clusters from the 303 clusters selected for participation in the 2011 Somaliland MICS [2]. The 2011 Somaliland MICS provided GPS coordinates for the approximate centroid of each of the 30 selected clusters, giving a useful starting point for mapping and listing each of the clusters. A mapping and listing exercise was conducted in each of the 30 clusters. The field staff used the centroid point from the Google Earth map as the starting location and moved in a clockwise direction sketching and listing up to 200 structures. The probability of selection of each household to be surveyed within each cluster was determined by dividing the total number of households in the selected cluster by the required 27 households to be reached; thus giving the interval of selection (n). The team then randomly selected a number within the interval which was identified as the "start" household. The remaining 26 households were selected using the interval to systematically select every nth household. All children 0 - 59 months of age in each selected household were eligible for participation in the survey.

\subsection{Ethical Considerations}

RINPS was a collaborative study between the Somaliland Ministry of Health (MOH), WHO and UNICEF Somalia offices with technical support from UNICEF/New York and the U.S. Centers for Disease Control and Prevention. The protocol was approved by a technical committee composed of the Somaliland MOH, UNICEF (Somalia) and WHO (Somalia). Coordination of the survey was guided by a steering committee. The steering committee established two technical committees-a coordination 
committee in Nairobi and an implementing committee in Somaliland. The steering committee met with the technical committees to review the study design, questionnaire, field procedures, data management, and analysis of RINPS. Permission to conduct RINPS was granted by the Somaliland $\mathrm{MOH}$ in November 2012. After approval from the Somaliland $\mathrm{MOH}$, ethical approval was obtained from local authorities in the regions and districts which included the selected RINPS clusters. In addition, for each household included, detailed information about the study was provided to the caregivers and oral consent was obtained before the interview was conducted.

\subsection{Data Analysis}

A three step weighting process was followed for RINPS. The first step consisted of calculating the base weight comprised of two factors: the probability of selection of the cluster and probability of selection of the household. The second step consisted of calculating non-response adjustments at the household and children levels. The third step consisted of calibrating the gender by age (0 11 months; 12 - 23 months; 24 - 35 months; 36 - 47 months; and 48 - 59 months) distribution from RINPS to match that of the 2006 Somaliland MICS. The 2006 MICS [3] distribution was used as the 2011 MICS had not been released at the time of the RINPS weighting process.

Data analysis was performed using STATA v12 statistical software procedures for analysis of complex survey data [4]. Weighted percentages are reported along with asymmetrical 95\% confidence intervals and un-weighted cell sample sizes. Any estimate with a denominator $<30$ was considered unreliable and not reported. Classifications of urban and rural were not used, however, analyses are presented based on the cluster location as either Hargeisa - the city serving as the capital for Somaliland and the second largest city in Somalia behind Mogadishuor the rest of Somaliland (i.e., all areas outside of Har- geisa).

\section{Results}

Of the 30 clusters selected, one (Sanaag Region, Laasqoray District) did not participate because of security issues. For the 29 participating clusters, 782 households were selected and 767 participated following consent provided by an adult in the household; in 15 households no one was at home. The household response rate was 98\%. In total, 883 children 0 - 59 months of age were identified in the 767 completed households. Of the 883 children, 867 participated, 14 were not at home, and the caregivers of 2 children refused. The interviewee response rate was $98 \%$. The overall response rate was $96 \%$.

\subsection{Participation in CHD and RIS}

Nearly 6 in 10 (58\%) children aged 0 - 59 months residing in Somaliland (at the time of the survey) participated in the October 2012 CHD (Table 1). The participation rate was similar for boys and girls. Participation was lower in Hargeisa (44\%) than the rest of Somaliland (69\%).

Caregivers of almost two-thirds of children aged 0 - 59 months (64\%) reported that the children had been vaccinated through routine immunization services (RIS) during their lifetime (Table 1). This percentage was similar for girls and boys, and was higher for children residing in Hargeisa (73\%) than the rest of Somaliland (57\%).

\subsection{Reason for Non-Participation in October 2012 CHD}

Among those children aged 0 - 59 months who did not participate in the October 2012 CHD, the most frequently cited reasons in Hargeisa were: lack of information (44\%) and lack of motivation (25\%) (Table 2). In addition, 12\% of children did not participate due to obstacles. Outside of Hargeisa, lack of motivation (37\%) was the most frequently cited reason given followed by obstacles (26\%) and lack of information (25\%).

Table 1. Participation in Child Health Days (CHD) and Routine Immunization Services (RIS) among children aged 0 - 59 months in Somaliland by sex and location-2012 Somaliland RINPS.

\begin{tabular}{|c|c|c|c|}
\hline $\begin{array}{l}\text { Child } \\
\text { Characteristic }\end{array}$ & $\begin{array}{c}\text { Participated in October } 2012 \\
\text { Child Health Day }\end{array}$ & $\begin{array}{l}\text { Ever received vaccination through } \\
\text { routine immunization system }\end{array}$ & Sample Size \\
\hline Total & $57.7(48.9,66.1)$ & $64.4(56.4,71.6)$ & 867 \\
\hline \multicolumn{4}{|l|}{ Sex } \\
\hline Male & $57.2(48.4,65.6)$ & $63.4(56.0,70.2)$ & 455 \\
\hline Female & $58.3(48.2,67.8)$ & $65.4(55.4,74.2)$ & 412 \\
\hline \multicolumn{4}{|l|}{ Area of residence } \\
\hline Hargeisa & $44.5(30.6,59.4)$ & $72.8(61.3,81.9)$ & 420 \\
\hline Outside of Hargeisa & $68.8(59.0,77.1)$ & $57.4(46.3,67.8)$ & 447 \\
\hline
\end{tabular}

Reported data are percentages; 95\% confidence interval reported in parentheses. 
Table 2. Reasons for non-participation in the October 2012 Child Health Day (CHD) among caregivers of 352 children aged 0 - 59 months in Somaliland—2012 Somaliland RINPS.

\begin{tabular}{|c|c|c|c|c|c|}
\hline \multirow{2}{*}{ Reported reason for not participating } & \multirow[b]{2}{*}{ Total } & \multicolumn{2}{|c|}{ Sex of child } & \multicolumn{2}{|c|}{ Area of residence } \\
\hline & & Boy & Girl & Hargeisa & Outside of Hargeisa \\
\hline Obstacles & $17.7(10.4,28.5)$ & $16.5(8.8,28.7)$ & $19.1(11.3,30.4)$ & $11.8(7.1,19.0)$ & $26.3(12.2,47.8)$ \\
\hline Place of CHD too far & $6.3(3.0,12.8)$ & $5.1(1.5,15.7)$ & $7.5(3.2,16.5)$ & $3.9(1.1,13.4)$ & $9.6(4.0,21.5)$ \\
\hline Child ill at time of CHD & $2.5(1.1,5.5)$ & $3.7(1.4,9.6)$ & $1.2(0.9,1.6)$ & $2.2(0.6,8.4)$ & $2.9(1.4,6.0)$ \\
\hline Long waiting line & $3.3(1.5,6.9)$ & $4.2(1.3,12.7)$ & $2.2(0.8,6.0)$ & $1.3(0.4,4.1)$ & $6.1(2.3,14.9)$ \\
\hline Time of CHD inconvenient & $2.6(0.9,7.9)$ & $1.9(0.7,5.2)$ & $3.5(0.8,13.9)$ & $1.4(0.3,6.0)$ & $4.5(1.1,16.7)$ \\
\hline Family problem & $3.1(1.1,8.6)$ & $1.5(0.3,7.1)$ & $4.8(1.9,11.5)$ & $2.9(0.9,9.0)$ & $3.2(0.5,19.5)$ \\
\hline Lack Motivation & $29.8(20.7,40.8)$ & $31.4(20.9,44.2)$ & $28.0(19.4,38.7)$ & $24.9(14.2,39.9)$ & $36.9(22.7,53.8)$ \\
\hline Mother too busy & $18.4(12.1,27.1)$ & $19.5(12.2,29.6)$ & $17.2(10.2,27.4)$ & $15.8(8.3,27.9)$ & $22.1(12.3,36.4)$ \\
\hline Cultural/Religious reasons & $8.8(4.6,16.3)$ & $9.5(4.1,20.4)$ & $8.1(4.2,14.9)$ & $7.1(4.3,11.7)$ & $11.2(3.5,30.7)$ \\
\hline Postponed until another time & $2.6(0.9,7.5)$ & $2.4(1.0,5.7)$ & $2.8(0.6,12.0)$ & $1.9(0.2,13.6)$ & $3.6(1.2,10.3)$ \\
\hline Lack Information & $36.4(24.9,49.6)$ & $35.3(21.5,51.9)$ & $37.6(27.4,49.1)$ & $44.4(27.9,62.2)$ & $24.8(13.8,40.5)$ \\
\hline Unaware of need to go to CHD & $18.5(12.1,27.1)$ & $16.7(9.7,27.1)$ & $20.4(12.9,30.8)$ & $21.7(12.8,34.3)$ & $13.8(7.3,24.5)$ \\
\hline Fear of side effects & $2.1(0.9,5.3)$ & $1.1(0.2,4.7)$ & $3.3(1.3,8.3)$ & $1.5(0.5,4.5)$ & $3.1(0.8,11.7)$ \\
\hline Place and/or time of CHD unknown & $15.8(8.8,26.7)$ & $17.6(9.2,30.9)$ & $13.9(7.9,23.3)$ & $21.2(10.7,37.9)$ & $8.0(3.3,18.3)$ \\
\hline Other & $16.1(11.0,22.8)$ & $16.9(11.5,24.0)$ & $15.2(9.3,23.8)$ & $18.9(12.3,28.0)$ & $11.9(5.7,23.3)$ \\
\hline Child not yet born at time of CHD & $5.3(2.4,11.7)$ & $5.7(2.5,12.5)$ & $5.0(1.8,12.8)$ & $5.5(1.6,17.1)$ & $5.2(2.2,11.9)$ \\
\hline Other & $10.7(7.7,14.7)$ & $11.2(7.9,15.5)$ & $10.2(6.2,16.4)$ & $13.5(10.2,17.6)$ & $6.8(2.7,16.0)$ \\
\hline Total & 100.0 & 100.0 & 100.0 & 100.0 & 100.0 \\
\hline Sample Size & $352^{*}$ & 193 & 159 & 218 & 134 \\
\hline
\end{tabular}

*11 observations have missing data for underlying reason child did not participate in the CHD Reported data are percentages; 95\% confidence interval reported in parentheses.

\subsection{Reasons for Not Receiving Immunizations through RIS}

The most common reasons for not having received routine vaccination through RIS in Hargeisa were: lack of motivation (50\%), followed by lack of information (29\%) (Table 3). Only 13\% reported obstacles as a reason for never having received routine vaccination through RIS (8\% vaccine not available). Outside of Hargeisa, the most common reasons for not having received a vaccination through RIS were: lack of motivation (36\%) and lack of information (34\%). Obstacles (20\%) were important outside of Hargeisa, especially place of vaccination too far $(16 \%)$.

\subsection{CHD Card Availability}

More than $90 \%$ of children who participated in the October 2012 CHD reportedly had received a CHD card; however, only $27 \%$ of the children had cards available to be seen by the enumerator (Table 4). There was no difference in card possession between male and female children, but possession was higher for those residing outside of Hargeisa (31\%) than within Hargeisa (21\%). Among those whose CHD card (either from the October 2012 CHD or a prior CHD) was not seen at the time of the interview, the most common reasons given were that the card was lost $(56 \%)$ or misplaced $(17 \%)$ or was damaged or destroyed (20\%).

\subsection{RIS Vaccination Card Availability}

During the survey, enumerators used sample routine vaccination cards to help respondents differentiate between the routine cards and those used in CHDs. A routine vaccination card through RIS was reportedly received by two-thirds of children at any time during their life (67\%); similar for girls and boys but higher for those residing in Hargeisa (75\%) compared with children residing outside of Hargeisa (60\%). However, at the time of the interview a routine vaccination card was available for examination for only $14 \%$ of children (Table 4 ). The cards were more often available for children residing in Hargeisa (18\%) than outside of Hargeisa (10\%).

\subsection{Vaccinations Received through CHD and/or RIS}

In Hargeisa, 81\% of children aged 0 - 59 months had received at least one routine vaccination (diphtheria-tetanus-pertussis [DTP] vaccine, oral poliovirus [OPV] vaccine and/or measles-containing vaccine [MCV]) in their lifetime either through CHDs, RIS, or both (Table 5). More than $40 \%$ had received at least one dose of DTP, OPV and/or MCV through RIS only; 5\% through CHD only; and 19\% had not been vaccinated for DTP, OPV, or MCV through either CHD or RIS. Outside of Hargeisa, $84 \%$ of children aged 0 - 59 months had received at least one routine vaccination in their lifetime from CHDs and/ 
Table 3. Reason for not receiving routine vaccination among children aged 0 - 59 months in Somaliland who report having never received routine vaccination through Routine Immunization Services-2012 Somaliland RINPS.

\begin{tabular}{|c|c|c|c|c|c|}
\hline \multirow{2}{*}{ Reason did not receive a vaccination } & \multicolumn{5}{|c|}{ Child Characteristic } \\
\hline & Total & Boy & Girl & Hargeisa & Outside of Hargeisa \\
\hline Obstacles & $16.9(9.6,27.9)$ & $16.2(8.2,29.6)$ & $17.7(8.1,34.2)$ & $12.9(4.0,34.2)$ & $19.9(10.8,33.7)$ \\
\hline Vaccine not available & $5.1(1.5,15.1)$ & $3.5(1.0,11.3)$ & $6.8(1.9,21.6)$ & $8.0(1.8,29.4)$ & $2.7(0.5,12.3)$ \\
\hline Place of vaccination too far & $11.0(5.4,21.1)$ & $11.7(5.3,23.7)$ & $10.1(3.1,28.4)$ & $4.3(0.7,21.9)$ & $16.2(8.0,30.0)$ \\
\hline Vaccinator absent & $1.9(0.4,8.7)$ & 0.0 & $4.3(0.9,18.1)$ & $4.4(0.9,19.2)$ & 0.0 \\
\hline Time of vaccination inconvenient & $0.6(0.0,4.6)$ & $1.1(0.1,8.0)$ & 0.0 & 0.0 & $1.0(0.1,8.2)$ \\
\hline Lack of motivation & $42.4(33.0,52.3)$ & $42.6(32.1,53.8)$ & $42.1(27.8,58.0)$ & $50.1(36.9,63.2)$ & $36.5(25.3,49.5)$ \\
\hline Mother too busy & $22.4(13.6,34.5)$ & $19.6(12.2,30.6)$ & $25.3(13.6,42.3)$ & $24.1(13.6,38.9)$ & $21.0(9.3,40.8)$ \\
\hline Cultural/religious reasons & $13.0(6.4,24.7)$ & $16.2(7.2,32.7)$ & $9.2(3.8,20.7)$ & $12.9(3.6,37.0)$ & $13.1(5.8,27.0)$ \\
\hline Postponed until another time & $6.2(2.6,13.8)$ & $6.5(1.8,21.2)$ & $5.8(2.4,13.2)$ & $10.9(4.3,25.1)$ & $2.4(0.3,18.2)$ \\
\hline Lack of information & $31.7(22.8,42.3)$ & $33.5(22.7,46.4)$ & $29.5(17.0,46.1)$ & $29.0(19.9,40.2)$ & $33.8(20.6,50.1)$ \\
\hline Unaware of need for immunization & $10.3(5.4,19.0)$ & $9.8(3.8,23.0)$ & $11.0(4.9,23.0)$ & $14.0(6.5,27.5)$ & $7.5(2.5,19.9)$ \\
\hline Fear of side effects & $3.0(0.8,11.0)$ & $1.8(0.2,15.4)$ & $4.4(0.9,19.9)$ & $2.0(0.2,17.6)$ & $3.8(0.7,17.9)$ \\
\hline Place and/or time of vaccination unknown & $5.8(1.9,16.4)$ & $3.5(1.2,9.7)$ & $8.4(2.4,25.5)$ & 0.0 & $10.3(3.9,24.6)$ \\
\hline Wrong ideas about contra-indications & $12.0(6.9,20.1)$ & $18.4(10.5,30.1)$ & $4.5(1.3,14.7)$ & $11.7(4.3,28.2)$ & $12.2(6.6,21.7)$ \\
\hline Don't Know & $8.8(3.87,19.3)$ & $7.7(4.0,14.2)$ & $10.3(3.1,28.7)$ & $7.7(1.8,27.3)$ & $9.8(3.4,25.2)$ \\
\hline Total & 100.0 & 100.0 & 100.0 & 100.0 & 100.0 \\
\hline Sample Size & $126^{*}$ & 72 & 54 & 59 & 67 \\
\hline
\end{tabular}

*168 observations missing data on underlying reasons for not having received routine vaccination. Reported data are percentages; 95\% confidence interval reported in parentheses.

Table 4. Child Health Day Card or Routine Vaccination Card availability at time of the RINPS—2012 Somaliland RINPS.

\begin{tabular}{lccc}
\hline Child Characteristic & $\begin{array}{c}\text { Child health day (CHD) } \\
\text { card available in home at time of RINPS }\end{array}$ & $\begin{array}{c}\text { Routine Immunization (RI) } \\
\text { card available in home at time of RINPS }\end{array}$ & $\begin{array}{l}\text { Sample Size } \\
\text { Total }\end{array}$ \\
Sex & $27.1(20.6,34.7)$ & $13.7(9.4,19.5)$ & 867 \\
Male & $26.0(20.6,32.3)$ & $12.7(9.0,17.6)$ & 455 \\
Female & $28.2(19.5,38.8)$ & $14.7(9.2,22.7)$ & 412 \\
Residence & & & 420 \\
Hargeisa & $21.4(12.6,34.1)$ & $18.1(10.8,28.8)$ & 447 \\
Outside of Hargeisa & $31.8(23.6,41.3)$ & $10.0(6.0,16.2)$ & \\
\hline
\end{tabular}

Reported data are percentages; 95\% confidence interval reported in parentheses. NOTE: CHD card refers to any CHD card ever received.

Table 5. Percentage of children aged 0 - 59 months residing in Somaliland who had received at least once dose of DTP, OPV, or MCV vaccine through Child Health Day (CHD) and/or routine immunization services (RIS) and the percentage who have never been vaccinated with these antigens through CHD or RIS - 2012 Somaliland RINPS.

\begin{tabular}{|c|c|c|c|c|c|c|}
\hline \multirow[b]{2}{*}{$\begin{array}{l}\text { Child } \\
\text { Characteristic }\end{array}$} & \multicolumn{3}{|c|}{ At least one dose of DTP, OPV, or MCV } & \multirow[b]{2}{*}{$\begin{array}{c}\text { Never vaccinated } \\
\text { through CHD or RIS }\end{array}$} & \multirow[b]{2}{*}{ Total } & \multirow[b]{2}{*}{ Sample Size } \\
\hline & $\begin{array}{l}\text { Both RIS } \\
\text { and CHD }\end{array}$ & RIS only & CHD only & & & \\
\hline Total & $\begin{array}{c}47.4 \\
(41.2,54.3)\end{array}$ & $\begin{array}{c}26.3 \\
(19.0,35.2)\end{array}$ & $\begin{array}{c}8.8 \\
(6.1,12.4)\end{array}$ & $\begin{array}{c}17.2 \\
(13.6,21.4)\end{array}$ & 100.0 & 867 \\
\hline Hargeisa & $\begin{array}{c}35.1 \\
(24.7,47.3)\end{array}$ & $\begin{array}{c}40.5 \\
(26.3,56.6)\end{array}$ & $\begin{array}{c}5.4 \\
(2.5,11.2)\end{array}$ & $\begin{array}{c}18.9 \\
(13.7,25.6)\end{array}$ & 100.0 & 420 \\
\hline Outside of Hargeisa & $\begin{array}{c}58.2 \\
(51.3,64.8)\end{array}$ & $\begin{array}{c}14.5 \\
(10.5,19.7)\end{array}$ & $\begin{array}{c}11.6 \\
(7.9,16.6)\end{array}$ & $\begin{array}{c}15.7 \\
(11.1,21.8)\end{array}$ & 100.0 & 447 \\
\hline
\end{tabular}

Reported data are percentages; 95\% confidence interval reported in parentheses. 
or RIS. However, unlike within Hargeisa, caregivers outside of Hargeisa were less likely to use RIS only ( $14 \%$ vs $40 \%$ ), but more likely to use CHD only ( $12 \%$ vs $5 \%$ ). Similar to within Hargeisa, $16 \%$ of children outside of Hargeisa had not been vaccinated for DTP, OPV, or MCV through either CHD or RIS.

\section{Discussion}

A recent assessment of CHDs in Somalia concluded their utilization is an effective strategy to achieve improvements in child survival and is important in settings such as Somalia [5]. The primary objective of the October 2012 CHD was to reach all children 0 - 59 months of age in Somalia, including all of Somaliland. Our findings from the Somaliland RINPS suggest fewer than 6 in 10 children participated in the October 2012 CHD. The participation in CHDs in the capital city of Hargeisa (44\%) and outside Hargeisa (68\%) is well below the target levels. It appears that social mobilization efforts regarding the October 2012 CHD may have been focused outside of Hargeisa due in part to an assumption about the performance of routine immunization services within Hargeisa. Of course, this represents a missed opportunity since one in five children in Hargeisa may have never received any immunizations.

It is seemingly important for CHDs to continue in Somaliland, at least in a targeted way, to prevent declines in immunization coverage given the gaps in delivery through traditional fixed site and outreach services. Based on our findings that only about $80 \%$ of children had received at least one dose of DTP, OPV or MCV (as a best case scenario for utilization of multi-dose antigens, since coverage for the second and third dose cannot exceed the levels for the first) and those from the 2011 Somaliland MICS report [2] and 2008 EPI coverage survey [6], Somaliland is well below internationally recommended levels of immunization coverage $[7,8]$. Moreover, RINPS results suggest that some children only access immunization services through CHDs (especially outside of Hargeisa) and that these same children would potentially be unreached if CHDs were to be discontinued. Stronger social mobilization efforts are needed throughout Somaliland, and especially in Hargeisa, to increase participation in CHDs given the apparent dependence on CHDs as a primary source of routine immunization for children.

Our findings of low RIS card retention are similar to those from the 2011 MICS, where only 20\% of children had routine immunization cards available at the time of the survey [2]. Overall, the prevalence of routine vaccination cards in Somaliland is unacceptably low, and it is clear that much is needed by way of 1) assuring cards are available for distribution, 2) improving communication from the Somaliland $\mathrm{MOH}$ and partners to health care professionals regarding issuance and utilization of cards and 3) improving communication from health care professionals to caregivers concerning the importance of keeping both routine and CHD vaccination cards, and bringing those cards with them to every CHD or RIS visit.

\section{Limitations}

The RINPS did not include known nomadic settlements; therefore, the prevalence of unreached children observed here is likely an underestimate. It should be noted, the Somaliland RINPS did not include specific questions on participation in recent polio focused National Immunization Days; thus, how effective this strategy is for boosting polio coverage is beyond the scope of this survey. The values reported in RINPS for children having received at least one dose of polio vaccine come largely from caregiver's recall and to a limited extent from CHD and routine immunization vaccination cards; whether caregivers are recalling doses of polio received through campaigns or other supplementary immunization activities as opposed to through routine immunization services cannot be determined here. Because about $80 \%$ of children reportedly received at least one dose of DTP, polio or measles vaccine through either CHDs or routine immunization; the sample sizes available for examination of reasons for non-participation were small and therefore estimates of sampling variance are large in some instances.

In conclusion, participation in the October 2012 CHD in Somaliland was well below the target level. The availability and delivery of sustainable routine immunization services need to be strengthened in Somaliland with a focus on strong social mobilization program to raise awareness about the importance of routine immunization and improve access points, particularly in areas outside of Hargeisa. The Somaliland MOH might consider an evaluation of the location of their maternal and child health clinics that offer immunizations and adjust as necessary. Finally, there are severe deficiencies in the support and maintenance of a basic medical record for children as reflected in the prevalence of home-based vaccination records [9]. Much more attention and resource investment are needed to improve recording and monitoring of vaccinations received in Somaliland, particularly as more expensive vaccines, such as pentavalent DTP-Hepatitis B-Haemophilus influenzae Type B vaccine, are introduced in Somalia.

\section{Acknowledgements}

The authors gratefully acknowledge Ms Sicily Matu, who served as the UNICEF/Somalia MICS Survey Coordinator for the 2011 Somaliland MICS, for her invaluable insights and technical support to this project. The 
authors would also like to acknowledge the RINPS field team supervisors, enumerators, and data entry staff. Finally, the authors would like to recognize the technical assistance received from the US Centers for Disease Control and Prevention, in particular Dr Charles Warren, Distinguished Fellow/Statistician, Centers for Disease Control and Prevention, CDC Emergency Operations Center, Polio Response.

\section{Disclaimer}

The findings and views expressed herein are those of the authors alone and do not necessarily reflect those of their respective institutions. Also, for the purposes of this survey, the analysis and reporting refers to Somaliland (also known as the Northwest Zone) according to the prewar zonal boundaries and does not imply any recognition of administrative boundaries by the United Nations.

\section{REFERENCES}

[1] WHO and UNICEF, "Estimates of National Immunization Coverage," Somalia Country Report, 2012 revision (completed July 2013), www.childinfo.org/files/Somalia_2000_2012.pdf

[2] UNICEF, "Preliminary Results 2011 Multiple Indicator Survey-Somaliland,” United Nations Children's Fund, 3
United Nations Plaza, New York. www.childinfo.org/mics4.html

[3] UNICEF, "Multiple Indicator Survey-Somalia. United Nations Children's Fund, 3 United Nations Plaza, New York, 2006. www.childinfo.org

[4] STATA Version 12, StataCorp LP, 4905 Lakeway Drive, College Station.

[5] I.R. Mirza, R. Kamadjeu, K. Assegid and A. Mulugeta, "Somalia: Supporting the Child Survival Agenda When Routine Health Service is Broken," The Journal of Infectious Diseases, Vol 205, Suppl. 1, 2012, pp. S126-S133. http://dx.doi.org/10.1093/infdis/jir776

[6] UNICEF/Somalia, "Somaliland Immunization Coverage Survey-July 2008,” UNICEF Somalia Support Center, Nairobi, Kenya.

[7] Decade of Vaccines Collaboration, "Decade of Vaccines-Global Vaccine Action Plan,” 2012. http://www.who.int/immunization/global_vaccine action _plan/DoV_GVAP_2012_2020/en/

[8] United Nations Children's Fund and World Health Organization, "Global immunization vision and strategy," 2005. http://whqlibdoc.who.int/hq/2005/WHO_IVB_05.05.pdf

[9] D. W. Brown, "Child Immunization Cards: Essential Yet Underutilized in National Immunization Programmes," The Open Vaccine Journal, Vol. 5, 2012, pp. 1-7. http://dx.doi.org/10.2174/1875035401205010001 